\title{
ERROR ESTIMATES FOR DISCRETIZED GALERKIN AND COLLOCATION BOUNDARY ELEMENT METHODS FOR TIME HARMONIC DIRICHLET SCREEN PROBLEMS IN $\mathbb{R}^{3}$
}

\author{
F. PENZEL \\ Fachbereich Mathematik, Technische Hochschule Darmstadt \\ D-6100 Darmstadt, Germany
}

1. Introduction. There is a series of papers on the analysis of the Galerkin and improved Galerkin methods for the boundary integral equation for the single layer potential for the Laplacian on the square plate $[10,11,12,20]$. Here we are interested in the error analysis of the boundary element discretization of time harmonic scalar problems which appear in direct acoustic scattering problems. The classical theory of these scattering problems for smoothly bounded exterior (or interior) domains is well described in [1]. The method of integral equations of the first kind and its rigorous theory for domains of that kind was initiated in [17, 15]. The underlying analysis for boundary integral equations and their approximation by Galerkin's method for screen problems in $\mathbb{R}^{3}$ using Sobolev spaces of locally finite energy was founded in [29]. Results for the transient acoustic scattering problem by flat objects have recently been obtained in [13]. A review of boundary integral equations is given in [32].

It is obvious that the computer implementation even of the Galerkin method has to be done by approximation of the scalar products, which results in additional error terms in the calculated solution. The same observation holds for the collocation method.

The question of convergence of collocation methods for general pseudodifferential operators on manifolds in $\mathbb{R}^{3}$ is still open. Several results were obtained recently, mainly for regular meshes and tensor-product splines. We refer the reader to $[27,4,3,23,24,5]$.

For one-dimensional boundary integral equations there is a lot of literature on

1991 Mathematics Subject Classification: 65R20.

The paper is in final form and no version of it will be published elsewhere. 
discretized Galerkin methods for singular integral equations [22] as for the single layer potential [14] and for general pseudo-differential operators [31]. A review on this field is given in [21].

Quadrature methods for the double layer potential equation (a singular integral equation of the second kind) over the boundary of a polyhedron have recently been extensively studied in [25].

A discretized Galerkin method for the boundary integral equation of the first kind on a square for the single layer potential of the Laplacian was analyzed in [18]. For practical use it was proved that it is sufficient to use a fixed number of calculations per matrix element to ensure convergence of the discretized method. The method was introduced by approximation of the scalar products of a Galerkin method by a composite Simpson-product formula. This formula was directly applied to integrals which have continuous integrands, while the weakly singular integrals are reduced to continuous ones by Duffy's [9] transformation of coordinates. Duffy's transformation of coordinates and its application in the theory of boundary integral equations are studied in [28].

In this paper we present a new result even for the Laplacian by defining a discretized method which uses only a one-point quadrature rule for "most" of the matrix elements. Our numerical approximation schemes are defined in Section 2 .

It is known that the boundary pseudodifferential operators for the Helmholtz equation are smooth and compact perturbations of the corresponding ones for the Laplacian [29]. By the methods of [13] we prove that the Galerkin equations here are uniquely solvable for all mesh widths (see Theorem 4.2(i) below). The same result is valid for the collocation methods, which follows from results in $[4,5]$. The unique solvability for all mesh widths does not in general carry over to the case of discretized Galerkin methods. The parameter dependence of the stability of these methods on the wave number and the chosen quadrature methods is presented in Theorem 4.4. More detailed estimates of the parameters can be found in [19]. In Section 5 we present our main result, in Theorem 5.2. There conditions on the parameters in the definition of our fully discretized schemes are given, to ensure convergence of our discretized schemes.

In Section 6 we present numerical calculations for the Laplacian to demonstrate that the low quadrature formula leads to good results.

2. Definition of numerical schemes. We assume $\Omega$ to be a bounded Lipschitz domain which is a subset of the square $[-1,1] \times[-1,1]$, such that the boundary of $\Omega$ has at least one point in common with the boundary of $[-1,1] \times$ $[-1,1]$. If $f$ is a distribution in $S^{\prime}\left(\mathbb{R}^{2}\right)$ we define the Fourier transform of $f$ by

$$
\widehat{f}(\xi):=\int_{\mathbb{R}^{2}} e^{i \xi \cdot x} f(x) d x \quad\left(\xi \in \mathbb{R}^{2}\right) .
$$


For given $s \in \mathbb{R}$ and $f, g \in S^{\prime}\left(\mathbb{R}^{2}\right)$ we define scalar products

$$
\langle f, g\rangle_{s}:=\int_{\mathbb{R}^{2}} \widehat{f}(\xi) \widehat{\widehat{g}(\xi)}(1+|\xi|)^{2 s} d \xi
$$

and norms

$$
\|f\|_{H^{s}\left(\mathbb{R}^{2}\right)}^{2}:=\langle f, f\rangle_{s}
$$

The closure of the space of infinitely differentiable functions with compact support in $\mathbb{R}^{2}, C_{0}^{\infty}\left(\mathbb{R}^{2}\right)$, with respect to this norm is the Sobolev space $H^{s}\left(\mathbb{R}^{2}\right)$. The closure of the space of infinitely differentiable functions with compact support in $\Omega, C_{0}^{\infty}(\Omega)$, with respect to this norm is the Sobolev space $\widetilde{H}^{s}(\Omega)$. In its dual space $H^{-s}(\Omega)$ we use (as usual) the norm

$$
\|f\|_{H^{-s}(\Omega)}:=\inf _{l f \in H^{-s}\left(\mathbb{R}^{2}\right)}\|l f\|_{H^{-s}\left(\mathbb{R}^{2}\right)},
$$

where the infimum is taken over all distributions $l f$ whose restriction to $\Omega$ coincides with $f$. In $L^{2}(\Omega)$ we also use the scalar product

$$
(f, g):=\int_{\Omega} f(x) \overline{g(x)} d x
$$

which is related to the already defined one by Plancherel's formula

$$
(f, g)=(2 \pi)^{-2}\langle f, g\rangle_{0} \text {. }
$$

We introduce the integral operators coming from the single layer potentials for the Helmholtz equation with wave number $\kappa \in \mathbb{C}$ :

$$
V_{\kappa} u(y):=\int_{\Omega} u(x) \Phi(x-y, \kappa) d x \quad(y \in \Omega),
$$

where $\Phi$ is (up to a constant) the fundamental solution for the Helmholtz equation defined by

$$
\Phi(x, \kappa):=\frac{e^{i \kappa|x|}}{|x|} .
$$

We shall assume that the wave number $\kappa$ has non-negative imaginary part $\kappa_{I}$. The function defined by (2.2) represents an outgoing wave [1]. We denote the real part of $\kappa$ by $\kappa_{R}$. We also use the operator

$$
\widetilde{V}_{\kappa} u(y):=\frac{1}{4 \pi} \int_{\Omega} u(x) \Phi(x-y, \kappa) d x \quad(y \in \Omega) .
$$

For a positive definite $M \times M$ matrix $A$ we denote by $\lambda_{\min }(A)\left(\lambda_{\max }(A)\right)$ the smallest (largest) eigenvalue of $A$. For $M \times M$ matrices $A$ we introduce norms by

$$
\|A\|_{\infty}:=\max _{i=1, \ldots, M} \sum_{k=1}^{M}\left|A_{i, k}\right|, \quad\|A\|_{2}:=\sqrt{\lambda_{\max }\left(A A^{*}\right)},
$$

where $A^{*}$ is the complex conjugate of the transpose of $A$. 
We now formulate the boundary integral equation in question [10, 12]:

$$
\widetilde{V}_{\kappa} w^{(\kappa)}(y)=\frac{1}{4 \pi} \int_{\Omega} w^{(\kappa)}(x) \Phi(x-y, \kappa) d x=f(y) \quad \text { for } y \in \Omega .
$$

$w^{(\kappa)}$ gives the jump of the normal derivative of the solution of Helmholtz's equation in $\mathbb{R}^{3} \backslash \bar{\Omega}$, which satisfies the Dirichlet problem with given $f$ on $\Omega$, and Sommerfeld's radiation condition $[1$, p. $70,(3.7)]$. Instead of $(2.3)$ we shall investigate the equivalent integral equation

$$
V_{\kappa} u^{(\kappa)}(y)=\int_{\Omega} u^{(\kappa)}(x) \Phi(x-y, \kappa) d x=f(y) \quad \text { for } y \in \Omega
$$

to simplify some constants. We look for functions $u^{(\kappa)} \in \widetilde{H}^{-1 / 2}(\Omega)$, given $f \in$ $H^{1 / 2}(\Omega)$. To discretize equation (2.3) we introduce for given $N \in \mathbb{N}$ the grids

$$
\square^{N}=\left\{x_{k}^{N}=\frac{k}{N}=\left(\frac{k_{1}}{N}, \frac{k_{2}}{N}\right) \mid k \in \mathbb{Z}^{2}\right\}
$$

and the functions $\varphi_{0}^{0}$ as the characteristic function of the interval $[0,1]$, and we let $\varphi_{0}^{1}(t)=\int_{\mathbb{R}} \varphi_{0}^{0}(x) \varphi_{0}^{0}(x-t) d x=\max (1-|t|, 0)$ be the normalized linear spline with node at $t_{0}=0$. For $d \in\{0,1\}$ the normalized tensor product splines are $\varphi_{0}^{0, d}\left(x_{1}, x_{2}\right)=\varphi_{0}^{d}\left(x_{1}\right) \varphi_{0}^{d}\left(x_{2}\right)$. On the grids $\square^{N}$ we introduce the spline functions of degree $d=0$ and $d=1$ by

$$
\varphi_{k}^{N, d}(x)=\varphi_{0}^{1, d}\left(N\left(x-x_{k}^{N}\right)\right) .
$$

In $\Omega$ we furthermore introduce the grids

$$
\omega^{N, d}=\left\{k=\left(k_{1}, k_{2}\right) \in \mathbb{Z}^{2} \mid \operatorname{supp}\left(\varphi_{k}^{N, d}\right) \subseteq \bar{\Omega}\right\} .
$$

$\omega^{N, d}$ is a totally ordered set if we use the lexicographical order

$$
k \leq k^{\prime}: \Leftrightarrow\left(k_{2}<k_{2}^{\prime} \text { or }\left(k_{2}=k_{2}^{\prime} \text { and } k_{1} \leq k_{1}^{\prime}\right)\right) \text {. }
$$

We define

as parameter for the mesh length.

$$
h:=\frac{1}{N}
$$

The Galerkin equations for (2.3) with piecewise constant trial functions then read

$$
\begin{aligned}
\int_{\Omega} \int_{\Omega} \sum_{k \in \omega^{N}, 0} \alpha_{k}^{\kappa} \varphi_{k}^{N, 0}(x) \varphi_{k^{\prime}}^{N, 0}(y) \Phi & (x-y, \kappa) d x d y \\
& =\int_{\Omega} f(y) \varphi_{k^{\prime}}^{N, 0}(y) d y, \quad \text { for } k^{\prime} \in \omega^{N, 0} .
\end{aligned}
$$

The collocation equations for piecewise bilinear ansatz functions collocating in the nodes read

$$
\int_{\Omega} \sum_{k \in \omega^{N, 1}} \beta_{k}^{\kappa} \varphi_{k}^{N, 1}(x) \Phi\left(x-x_{k^{\prime}}^{N}, \kappa\right) d x=f\left(x_{k^{\prime}}^{N}\right), \quad \text { for } k^{\prime} \in \omega^{N, 1} .
$$


It is known $[12,29]$ that the Galerkin schemes lead to approximations $u_{h}^{(\kappa)}=$ $\sum_{k \in \omega^{N, 0}} \alpha_{k}^{\kappa} \varphi_{k}^{N, 0}$ of the uniquely determined solutions $u^{(\kappa)}$ of the integral equation (2.3) for all $f \in H^{1 / 2}(\Omega)$.

If one is interested in functions $f$ which also have higher regularity, say $f \in$ $H^{s}(\Omega), s>1$, then it is known $[12,7]$ that the solutions $u^{(\kappa)}$ of the integral equations are in $H^{-\varepsilon}(\Omega)\left(=\widetilde{H}^{-\varepsilon}(\Omega)\right)$ for any $0<\varepsilon<\frac{1}{2}$. Furthermore, the following quasi-optimal convergence result $[12,2,6]$ holds for the spline functions $u_{h}^{\kappa}$ which are defined by the Galerkin equations (2.5):

$$
\left\|u^{(\kappa)}-u_{h}^{\kappa}\right\|_{\widetilde{H}^{-1 / 2}(\Omega)} \leq C h^{1 / 2-\varepsilon}\|f\|_{H^{s}(\Omega)}
$$

with a constant $C$ independent of $f$ and $h$ (but depending on $\kappa$ ).

For $\kappa=0$ a similar result is derived for the solution $v_{h}^{(0)}=\sum_{k \in \omega^{N, 1}} \beta_{k}^{0} \varphi_{k}^{N, 1}$ of the collocation method in [5]. The case of $\kappa \neq 0$ can also be handled by the methods introduced in that paper.

The following lemma gives a representation of the coefficient matrices of the numerical schemes (2.4), (2.5).

LEMma 2.1. For $k, k^{\prime} \in \omega^{N, 0}$ we have

$$
A_{k, k^{\prime}}^{(\kappa, N)}:=\left(V_{\kappa} \varphi_{k}^{N, 0}, \varphi_{k^{\prime}}^{N, 0}\right)=h^{3} \int_{[-1,1]^{2}} \varphi_{0}^{1,1}(x) \Phi\left(x-k+k^{\prime}, h \kappa\right) d x
$$

and

$$
\left(V_{\kappa} \varphi_{k}^{N, 0}, \varphi_{k^{\prime}}^{N, 0}\right)=h^{2}\left(V_{\kappa} \varphi_{k}^{N, 1}\right)\left(x_{k^{\prime}}^{N, 0}\right)
$$

R e marks. 1. The representation (2.8) is proved as in [18, Lemma 2.1]. The equality of block Toeplitz matrices stated in (2.9) was shown in [4].

2. The matrix elements $\left(V_{\kappa} \varphi_{k}^{N, 0}, \varphi_{k^{\prime}}^{N, 0}\right)$ only depend on $\left(\left|k_{1}-k_{1}^{\prime}\right|,\left|k_{2}-k_{2}^{\prime}\right|\right)$. So we introduce matrices $B^{(\kappa, N)}$ by

$$
B_{k}^{(\kappa, N)}=h^{3} \int_{\mathbb{R}^{2}} \varphi_{0}^{1,1}(x) \Phi(x-k, h \kappa) d x=h^{3} \int_{[-1,1]^{2}} \varphi_{0}^{1,1}(x) \Phi(x-k, h \kappa) d x
$$

for $k \in \mathbb{N}_{0}^{2}, k \in[0,2 N]^{2}$. In the following we shall also denote by $B_{k}^{(\kappa, N)}$ the right-hand side of (2.10) for arbitrary $k \in \mathbb{Z}^{2}$.

Here we introduce a numerical scheme which depends on several parameters to discretize the sesquilinear forms which are defined by Galerkin's scheme (2.5) and the collocation scheme (2.6).

For a given continuous function $g:[0,1] \times[0,1] \rightarrow \mathbb{R}$ we introduce as in $[18]$ the composite Simpson-product formula of mesh length $1 / M$ : 


$$
\begin{aligned}
C S P(g) & :=\sum_{l=0}^{M-1} \sum_{m=0}^{M-1} \frac{1}{36 M^{2}}\left[g\left(\frac{m}{M}, \frac{l}{M}\right)+4 g\left(\frac{m}{M}, \frac{2 l+1}{2 M}\right)+g\left(\frac{m}{M}, \frac{l+1}{M}\right)\right. \\
& +4 g\left(\frac{2 m+1}{2 M}, \frac{l}{M}\right)+16 g\left(\frac{2 m+1}{2 M}, \frac{2 l+1}{2 M}\right)+4 g\left(\frac{2 m+1}{2 M}, \frac{l+1}{M}\right) \\
& \left.+g\left(\frac{m+1}{M}, \frac{l}{M}\right)+4 g\left(\frac{m+1}{M}, \frac{2 l+1}{2 M}\right)+g\left(\frac{m+1}{M}, \frac{l+1}{M}\right)\right] .
\end{aligned}
$$

There are three different types of integrals in the coefficient matrices characterized by singularities of their integrands. To approximate the values $\left(V_{\kappa} \varphi_{k}^{N, 0}, \varphi_{k^{\prime}}^{N, 0}\right)$ which are defined by integrals with singular integrands, we first use the formula

$$
\int_{[-1,1]^{2}} \varphi_{0}^{1,1}(x) \Phi(x-k, h \kappa) d x \approx \int_{[-1,1]^{2}} \frac{\varphi_{0}^{1,1}(x)}{|x-k|} d x,
$$

which is obtained from the Taylor expansion of the function $e^{z}$ around $z_{0}=0$ taking $z=i h \kappa|x-k|$. Next we approximate the integral $\int_{[-1,1]^{2}}\left(\varphi_{0}^{1,1}(x) /|x-k|\right) d x$ as in [18] by using Duffy's transformation of coordinates [9]

$$
u_{1} \rightarrow v_{1}, \quad u_{2} \rightarrow v_{1} v_{2} \text {. }
$$

This substitution transforms the triangle with corners $(0,0),(1,0),(1,1)$ into the rectangle with corners $(0,0),(0,1),(1,1),(1,0)$ and integrands with square-root singularities at $(0,0)$ into smooth integrands. This leads to the representation

$$
B_{(0,0)}^{0, N}=h^{3} \int_{[0,1]^{2}} 8 \frac{(1-t)(1-s t)}{\sqrt{\left(1+s^{2}\right)}} d s d t .
$$

Similar representations for $B_{(1,0)}^{0, N}, B_{(1,1)}^{0, N}$ are in $[18,19]$.

Our Galerkin methods and collocation methods define bilinear forms on the piecewise constant functions or the piecewise bilinear functions, respectively.

For $L, M \in \mathbb{N}$ we define discretized Galerkin and collocation methods by the introduction of matrices $q^{\kappa, L, M, N}$ given by

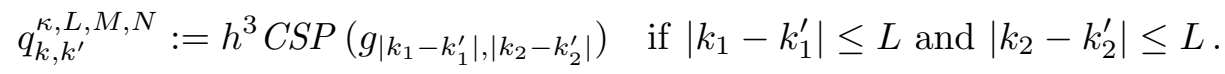

The functions $g_{k}$ were already defined for $k \in\{(0,0),(1,0),(0,1),(1,1)\}$. For other values of $k$, we define

$$
\begin{aligned}
& g_{k}\left(x_{1}, x_{2}\right) \\
& \quad:=\left(1-x_{1}\right)\left(1-x_{2}\right)\left(\sum_{m=1}^{2} \sum_{n=1}^{2} \Phi\left(\left(x_{1}+(-1)^{m} k_{1}, x_{2}+(-1)^{n} k_{2}\right), 0\right)\right) .
\end{aligned}
$$

Then by double symmetrization it is easy to see that the identity

$$
\left(V_{0} \varphi_{k}^{N, 0}, \varphi_{k^{\prime}}^{N, 0}\right)=\int_{0}^{1} \int_{0}^{1} g_{k-k^{\prime}}\left(x_{1}, x_{2}\right) d x_{1} d x_{2}
$$

holds. 
If $\left|k_{1}-k_{1}^{\prime}\right|>L$ or if $\left|k_{2}-k_{2}^{\prime}\right|>L$ then the matrix elements $q_{k, k^{\prime}}^{\kappa, L, M, N}$ are defined simply by

$$
q_{k, k^{\prime}}^{\kappa, L, M, N}:=h^{3} \Phi\left(\left(\left|k_{1}-k_{1}^{\prime}\right|,\left|k_{2}-k_{2}^{\prime}\right|\right), h \kappa\right)=h^{3} \frac{e^{i h \kappa\left|k-k^{\prime}\right|}}{\left|k-k^{\prime}\right|} .
$$

This approximation is found by application of the midpoint rule to the matrix elements $\left(V_{\kappa} \varphi_{k}^{N, 0}, \varphi_{k^{\prime}}^{N, 0}\right)$, given by (2.8). It will be proved that it coincides with the first term of the asymptotic expansion of that integral for $\left|k-k^{\prime}\right| \rightarrow \infty$.

Our discretized Galerkin schemes will be defined by the linear systems

$$
\sum_{k \in \omega^{N, 0}} \gamma_{k}^{\kappa} q_{k, k^{\prime}}^{\kappa, L, N}=h^{2} f\left(x_{k^{\prime}}^{N}\right), \quad \text { for } k^{\prime} \in \omega^{N, 0},
$$

which in the case of existence lead to the solution $u_{N}^{\kappa}:=\sum_{k \in \omega^{N, 0}} \gamma_{k}^{\kappa} \varphi_{k}^{N, 0}$. Here we use the midpoint formula for approximation of the right-hand side. We define our discretized Galerkin method for functions $f \in H^{2+\varepsilon}(\Omega)$ for $\varepsilon>0$, which implies continuous differentiability of $f$.

The discretized collocation schemes are introduced by the linear systems

$$
\sum_{k \in \omega^{N, 1}} N^{2} \varrho_{k}^{\kappa} q_{k, k^{\prime}}^{\kappa, L, M, N}=f\left(x_{k^{\prime}}^{N}\right), \quad \text { for } k^{\prime} \in \omega^{N, 1},
$$

which in the case of existence leads to the solution

$$
v_{N}^{\kappa}:=\sum_{k \in \omega^{N, 0}} \varrho_{k}^{\kappa} \varphi_{k}^{N, 1}
$$

3. Consistency. In this section we prove consistency estimates for the matrix elements of the discretized Galerkin and collocation methods introduced in Section 2.

Let us introduce the notation

$$
d(k):=\min _{x \in[-1,1]^{2}}|x-k|
$$

LEMMA 3.1. If $L>1, k \in \mathbb{N}_{0}^{2}, k_{1}>L$ or $k_{2}>L$ and $h>0, \kappa_{I} \geq 0$, $x \in[-1,1]^{2}$ then the following estimate holds:

$$
\begin{aligned}
\mid \int_{[-1,1]^{2}}(\Phi(x-k, h \kappa) & -\Phi(k, h \kappa)) \varphi_{0}^{1,1}(x) d x \mid \\
& =\left|\int_{[-1,1]^{2}} \Phi(x-k, h \kappa) \varphi_{0}^{1,1}(x) d x-\Phi(k, h \kappa)\right| \\
& \leq \frac{h^{2}|\kappa|^{2}}{24 d(k)}+\frac{h|\kappa|}{12 d^{2}(k)}+\frac{1}{12 d^{3}(k)} .
\end{aligned}
$$


Pr o of. The following representation holds for the partial derivatives of $\Phi$ :

$$
\begin{aligned}
\frac{\partial \Phi(x, h \kappa)}{\partial x_{1}}(x) & =\frac{i h \kappa|x|-1}{|x|^{2}} x_{1} \Phi(x, h \kappa), \\
\frac{\partial^{2} \Phi(x, h \kappa)}{\partial x_{1}^{2}}(x) & =\frac{-h^{2} \kappa^{2} x_{1}^{2}|x|^{2}-2 i h \kappa x_{1}^{2}|x|+i h \kappa x_{2}^{2}|x|+2 x_{1}^{2}-x_{2}^{2}}{|x|^{4}} \Phi(x, h \kappa), \\
\frac{\partial^{2} \Phi(x, h \kappa)}{\partial x_{1} \partial x_{2}}(x) & =\frac{\left(-h^{2} \kappa^{2}|x|^{2}-2 i h \kappa|x|+2\right) x_{1} x_{2}-(i h \kappa|x|-1) x_{1} x_{2}}{|x|^{4}} \Phi(x, h \kappa) .
\end{aligned}
$$

By Taylor's expansion of $\Phi(x-k, h \kappa)$ around $x=0$ we obtain

$$
\begin{aligned}
\int_{[-1,1]^{2}} \Phi(x-k, h \kappa) \varphi_{0}^{1,1}(x) d x & =\int_{[-1,1]^{2}} \Phi(k, h \kappa) \varphi_{0}^{1,1}(x) d x \\
& +\int_{[-1,1]^{2}} k_{1} x_{1} \frac{i h \kappa|k|-1}{|k|^{2}} \Phi(k, h \kappa) \varphi_{0}^{1,1}(x) d x \\
& +\int_{[-1,1]^{2}} k_{2} x_{2} \frac{i h \kappa|k|-1}{|k|^{2}} \Phi(k, h \kappa) \varphi_{0}^{1,1}(x) d x \\
+ & \frac{1}{2} \int_{[-1,1]^{2}} \int_{0}^{1} x^{\mathrm{T}} D^{2} \Phi(\vartheta x-k, h \kappa) x d \vartheta \varphi_{0}^{1,1}(x) d x
\end{aligned}
$$

where $x^{\mathrm{T}}$ is the transpose of $x$, and $D^{2} \Phi(x)$ is the matrix defined by

$$
D^{2} \Phi(x)=\left(\begin{array}{cc}
\frac{\partial^{2} \Phi}{\partial x_{1}^{2}}(x) & \frac{\partial^{2} \Phi}{\partial x_{1} \partial x_{2}}(x) \\
\frac{\partial^{2} \Phi}{\partial x_{1} \partial x_{2}}(x) & \frac{\partial^{2} \Phi}{\partial x_{2}^{2}}(x)
\end{array}\right) .
$$

Taking into account

$$
\int_{[-1,1]^{2}} \varphi_{0}^{1,1}(x) d x=1, \quad \int_{[-1,1]^{2}} x_{1} \varphi_{0}^{1,1}(x) d x=0=\int_{[-1,1]^{2}} x_{2} \varphi_{0}^{1,1}(x) d x
$$

we get

$$
\begin{aligned}
\mid \int_{[-1,1]^{2}} \Phi(x-k, h \kappa) \varphi_{0}^{1,1}(x) & d x-\Phi(k, h \kappa) \mid \\
\leq & \frac{1}{2} \int_{[-1,1]^{2}} \int_{0}^{1}\left|x^{\mathrm{T}} D^{2} \Phi(\vartheta x-k, h \kappa) x\right| d \vartheta \varphi_{0}^{1,1}(x) d x .
\end{aligned}
$$

So the essential point that the linear term in the Taylor remainder vanishes after integration comes out. By the explicit representation of the second derivatives, the asymptotic behaviour as $k \rightarrow \infty$ of the left-hand side of (3.1) is obvious. The constants are estimated in [19]. 
An estimate of the error $\left\|A^{(\kappa, N)}-q^{\kappa, L, M, N}\right\|_{\infty}$ will be derived later with the help of the following lemma.

LEMma 3.2. There are non-negative constants $C_{1}, C_{2}, C_{3}, C_{4}$ such that for all $N, L \in \mathbb{N}, h=1 / N, \kappa_{I} \geq 0, N>L \geq 2$, the following estimate holds:

$$
\begin{aligned}
\sum_{k \in[-2 N, 2 N]^{2} \backslash[-L, L]^{2}} \mid & \int_{[-1,1]^{2}} \Phi(x-k, h \kappa) \varphi_{0}^{1,1}(x) d x-\Phi(k, h \kappa) \mid \\
\leq & C_{1} h^{2}|\kappa|^{2} \ln N+C_{2} h|\kappa| \ln N \\
& +C_{3} h \frac{|\kappa|}{2(L-1)}+C_{4} h|\kappa|^{2}+\frac{1}{(L-1)^{2}}+\frac{\pi}{6(L-1)} .
\end{aligned}
$$

Pro of. From Lemma 3.1 we get

$$
\begin{aligned}
\sum_{k \in[-2 N, 2 N]^{2} \backslash[-L, L]^{2}} \mid & \int_{[-1,1]^{2}} \Phi(x-k, h \kappa) \varphi_{0}^{1,1}(x) d x-\Phi(k, h \kappa) \mid \\
\leq & \sum_{k \in[-2 N, 2 N]^{2} \backslash[-L, L]^{2}}\left(\frac{h^{2}|\kappa|^{2}}{24 d(k)}+\frac{h|\kappa|}{12 d^{2}(k)}+\frac{1}{12 d^{3}(k)}\right) .
\end{aligned}
$$

This sum can be estimated by comparison with an integral over a domain with diameter growing with $N$. Because of

$$
\int_{1}^{N} \int_{0}^{\pi / 2} r^{-n} r d \alpha d r=O\left(N^{2-n}\right), \quad N \rightarrow \infty
$$

the formula comes out. Again, a more detailed proof with determination of constants is presented in [19].

In the next lemma we state an estimate of $\left|\left(V_{\kappa} \varphi_{k}^{N, 0}, \varphi_{k^{\prime}}^{N, 0}\right)-q_{k, k^{\prime}}^{\kappa, L, N}\right|$ for given $k-k^{\prime} \in[-L, L]^{2}$.

Lemma 3.3. For $N, L \in \mathbb{N}, h=1 / N, \kappa_{I} \geq 0, N>L>1$, the following estimates hold.

(i) If $k-k^{\prime} \in[-L, L]^{2} \backslash[-1,1]^{2}$ then

$$
\left|\left(V_{\kappa} \varphi_{k}^{N, 0}, \varphi_{k^{\prime}}^{N, 0}\right)-q_{k, k^{\prime}}^{\kappa, L, N}\right| \leq h^{4}|\kappa|+\frac{3 h^{3}}{20 M^{4} d^{5}\left(k-k^{\prime}\right)}+\frac{h^{3}}{10 M^{4} d^{4}\left(k-k^{\prime}\right)} .
$$

(ii) If $k=k^{\prime}$ then

$$
\left|\left(V_{\kappa} \varphi_{k}^{N, 0}, \varphi_{k}^{N, 0}\right)-q_{k, k}^{\kappa, L, M, N}\right| \leq h^{4}|\kappa|+\frac{h^{3}}{12 M^{4}} .
$$

(iii) If $k-k^{\prime} \in[-1,1]^{2}, k \neq k^{\prime}$ then

$$
\left|\left(V_{\kappa} \varphi_{k}^{N, 0}, \varphi_{k^{\prime}}^{N, 0}\right)-q_{k, k^{\prime}}^{\kappa, L, M, N}\right| \leq h^{4}|\kappa|+\frac{h^{3}}{9 M^{4}} .
$$


P r o of of (i). First we compare the matrix elements corresponding to the single layer operator of the Helmholtz equation with their analogues for the Laplace equation:

$$
\begin{aligned}
\left|\left(V_{\kappa} \varphi_{k}^{N, 0}, \varphi_{k^{\prime}}^{N, 0}\right)-\left(V_{0} \varphi_{k}^{N, 0}, \varphi_{k^{\prime}}^{N, 0}\right)\right| & \leq h^{3} \int_{[-1,1]^{2}} \varphi_{0}^{1,1}(x)\left|\frac{e^{i h \kappa\left|x-k+k^{\prime}\right|}-1}{\left|x-k+k^{\prime}\right|}\right| d x \\
& =h^{3} \int_{[-1,1]^{2}} \varphi_{0}^{1,1}(x)\left|\int_{0}^{1} i h \kappa e^{i \vartheta h \kappa\left|x-k+k^{\prime}\right|} d \vartheta\right| d x \\
& \leq \int_{[-1,1]^{2}} \varphi_{0}^{1,1}(x) h^{4}|\kappa| d x=h^{4}|\kappa|
\end{aligned}
$$

We used the fact that the argument of the exponential function above always has non-positive real part.

So it is sufficient to estimate the error coming from the "static part" $\left|\left(V_{0} \varphi_{k}^{N, 0}, \varphi_{k^{\prime}}^{N, 0}\right)-q_{k, k^{\prime}}^{0, L, M, N}\right|$, taking into account that we defined $q_{k, k^{\prime}}^{0, L, M, N}=$ $q_{k, k^{\prime}}^{\kappa, L, N}$ under the given restrictions on the values of $k$ and $k^{\prime}$.

The following inequalities have been proved in [18, Lemma 3.3]:

$$
\left|\left(V_{0} \varphi_{k}^{N, 0}, \varphi_{k}^{N, 0}\right)-q_{k, k}^{0, L, M, N}\right| \leq \frac{h^{3}}{12 M^{4}}, \quad\left|\left(V_{0} \varphi_{k}^{N, 0}, \varphi_{k^{\prime}}^{N, 0}\right)-q_{k, k^{\prime}}^{0, L, M, N}\right| \leq \frac{h^{3}}{9 M^{4}} ;
$$

the latter holds under the assumption $k-k^{\prime} \in[-1,1]^{2}, k \neq k^{\prime}$.

If $k-k^{\prime} \notin[-1,1]^{2}$ then it is easily derived from [18, Lemma 3.2] that

$$
\begin{aligned}
\left|\left(V_{0} \varphi_{k}^{N, 0}, \varphi_{k^{\prime}}^{N, 0}\right)-q_{k, k^{\prime}}^{0, L, M, N}\right| & \leq \frac{h^{3}}{1440 M^{4}} 4\left(\frac{54}{d^{5}\left(k-k^{\prime}\right)}+\frac{36}{d^{4}\left(k-k^{\prime}\right)}\right) \\
& =\frac{3 h^{3}}{20 M^{4} d^{5}\left(k-k^{\prime}\right)}+\frac{h^{3}}{10 M^{4} d^{4}\left(k-k^{\prime}\right)}
\end{aligned}
$$

The proofs of the lemmas cited used the well-known error estimate for the composite Simpson product formula [8, p. 45/46], [30],

$$
\left|\int_{0}^{1} \int_{0}^{1} g(x, y) d x d y-C S P(g)\right| \leq \frac{\left\|\partial^{4} g / \partial x^{4}\right\|_{\infty}+\left\|\partial^{4} g / \partial y^{4}\right\|_{\infty}}{2880 M^{4}}
$$

which is available for $g \in C^{4}\left([0,1]^{2}\right)$. The asymptotic correctness of the error estimate (3.7) when $\left|k-k^{\prime}\right|$ tends to infinity already follows from the observation that the fourth derivatives with respect to $x$ of the restriction to $[0,1]^{2}$ of the function $\varphi_{0}^{1,1}(x) /\left|x-k+k^{\prime}\right|$ are of order $d^{-4}\left(k-k^{\prime}\right)$.

LEMMA 3.4. If $N, L \in \mathbb{N}, h=1 / N, \kappa_{I} \geq 0, N>L>1$, then

$$
\sum_{k \in[-L, L]^{2}}\left|\left(V_{\kappa} \varphi_{k}^{N, 0}, \varphi_{0}^{N, 0}\right)-q_{k, 0}^{\kappa, L, M, N}\right| \leq 4(L-1)^{2} h^{4}|\kappa|+5.1 \frac{h^{3}}{M^{4}} .
$$


Pr o of. The methods of the foregoing result can be used to prove the lemma, see $[19]$.

After these preparations we are in a position to estimate the difference of the matrices $A^{\kappa, N}$ defined by (2.8) and the matrices of our discretized methods $q^{\kappa, L, M, N}$, which are defined by $(2.13)$ and (2.16) measured in the $\|\cdot\|_{\infty}$-norm. In [14] the authors used the Schur norm to prove consistency of the matrix elements for one-dimensional problems.

THEOREM 3.5. There is a constant $C>0$ such that for all $N, L \in \mathbb{N}, h=1 / N$, $\kappa_{I} \geq 0$, the following consistency estimate holds:

$$
\begin{aligned}
\left\|A^{(\kappa, N)}-q^{\kappa, L, M, N}\right\|_{\infty} \leq & C\left(h^{5}|\kappa|^{2} \ln N+h^{4}|\kappa| \ln N+h^{4}|\kappa|(L-1)^{2}\right) \\
& +h^{3}\left(\frac{1}{2(L-1)^{2}}+\frac{\pi}{6(L-1)}\right)+5.1 \frac{h^{3}}{M^{4}} .
\end{aligned}
$$

Proof. Here we extend the definition of $A_{k, k^{\prime}}^{\kappa, N}-q_{k, k^{\prime}}^{\kappa, L, M, N}$ to $k \in \mathbb{Z}^{2}$ and $k^{\prime} \in \mathbb{Z}^{2}$ by means of the right-hand sides of (2.8), (2.16), respectively. Because of

$$
A_{k, k^{\prime}}^{\kappa, N}-q_{k, k^{\prime}}^{\kappa, L, M, N}=\left(V_{\kappa} \varphi_{\left|k-k^{\prime}\right|}^{N, 0}, \varphi_{0}^{N, 0}\right)-q_{\left|k-k^{\prime}\right|, 0}^{\kappa, L, M, N}
$$

it follows by the substitution $k^{\prime} \rightarrow k^{\prime \prime}=k-k^{\prime}$ that

$$
\begin{aligned}
\left\|A^{(\kappa, N)}-q^{\kappa, L, M, N}\right\|_{\infty} & =\max _{k \in[-N, N]^{2}} \sum_{k^{\prime} \in[-N, N]^{2}}\left|A_{\left|k-k^{\prime}\right|, 0}^{\kappa, N}-q_{\left|k-k^{\prime}\right|, 0}^{\kappa, L, M, N}\right| \\
& =\max _{k \in[-N, N]^{2}} \sum_{k_{1}^{\prime \prime}=k_{1}-N}^{k_{1}+N} \sum_{k_{1}^{\prime \prime}=k_{2}-N}^{k_{2}^{\prime \prime}+N}\left|A_{\left(\left|k_{1}^{\prime \prime}\right|,\left|k_{2}^{\prime \prime}\right|\right), 0}^{\kappa, N}-q_{\left(\left|k_{1}^{\prime \prime}\right|,\left|k_{2}^{\prime \prime}\right|\right), 0}^{\kappa, L, M, N}\right| \\
& \leq \sum_{k^{\prime \prime} \in[-2 N, 2 N]^{2}} \mid A_{\left(\left|k_{1}^{\prime \prime}\right|,\left|k_{2}^{\prime \prime}\right|\right), 0}^{\kappa, N}-q_{\left(\left|k_{1}^{\prime \prime}\right|,\left|k_{2}^{\prime \prime}\right|\right), 0}^{\kappa, L, M, N} .
\end{aligned}
$$

Using the estimates of Lemmas 3.3 and 3.4 we obtain the final result (3.9).

R e m a rk. Theorem 3.5 demonstrates that for a given wave number $\kappa$ satisfying $\kappa_{I} \geq 0$, there are absolute constants $C_{1}, C_{2}$ and $C_{3}$ such that

$$
\left\|A^{(\kappa, N)}-q^{\kappa, L, M, N}\right\|_{\infty} \leq C_{1} \frac{h^{3}}{M^{4}}+C_{2} \frac{h^{3}}{L-1}+C_{3} h^{4} L^{2} .
$$

So given $\varepsilon>0$ arbitrarily small we can (by choosing $L$ and $M$ sufficiently large) have the estimate

$$
\left\|A^{(\kappa, N)}-q^{\kappa, L, M, N}\right\|_{\infty} \leq \varepsilon h^{3}
$$

for sufficiently small $h$. This was proved in the case $\kappa=0$ in [18] to be sufficient to get convergence of the fully discretized scheme. The same will be proved for general $\kappa$ in Sections 4 and 5 . 
4. Stability. We reduce the question of invertibility of the matrices of the discretized methods to the invertibility of the matrices of the Galerkin scheme by a perturbation argument. The invertibility of the Galerkin matrices for all $\kappa_{I} \geq 0$ and all $N \in \mathbb{N}$ will be proved as well. The latter result was derived in [13]. We need some estimates for the inverses of the Galerkin matrices.

LEMMA 4.1. Denote by $\|\cdot\|$ any vector norm in $\mathbb{C}^{n} ;$ by the same symbol we denote a compatible matrix norm in $\mathbb{C}^{(n, n)}$. If $A$ is an invertible $n \times n$ matrix then

$$
\left\|A^{-1}\right\|^{-1} \geq \inf _{0 \neq x \in \mathbb{C}^{n}} \frac{|(A x, x)|}{\|x\|^{2}} .
$$

P r o of. By the definition of a compatible matrix norm, the Cauchy-Schwarz inequality and the substitution $y=A x$ we obtain

$$
\begin{aligned}
\left\|A^{-1}\right\| & =\sup _{y \neq 0} \frac{\left\|A^{-1} y\right\|}{\|y\|}=\sup _{y \neq 0} \frac{\left\|A^{-1} y\right\|^{2}}{\left\|A^{-1} y\right\|\|y\|} \\
& \leq \sup _{y \neq 0} \frac{\left\|A^{-1} y\right\|^{2}}{\left|\left(A^{-1} y, y\right)\right|}=\left(\inf _{y \neq 0} \frac{\left|\left(A^{-1} y, y\right)\right|}{\left\|A^{-1} y\right\|^{2}}\right)^{-1}=\left(\inf _{x \neq 0} \frac{|(x, A x)|}{\|x\|^{2}}\right)^{-1} .
\end{aligned}
$$

The following theorem contains estimates for the inverse norm of the Galerkin matrices for wave numbers $\kappa$ which satisfy $\kappa_{I} \geq 0$. This result was proved in [18, Lemma 4.2] in the special case $\kappa=0$.

THEOREM 4.2. (i) If $\kappa_{I} \geq 0$ then the matrices $A^{(\kappa, N)}$ are invertible for all $N \in \mathbb{N}$.

(ii) If $\kappa=0$ then

$$
\left\|\left(A^{(\kappa, N)}\right)^{-1}\right\|_{2}^{-1} \geq \frac{\pi}{5} h^{3}
$$

(iii) If $\kappa_{I}>0$ then

$$
\left\|\left(A^{(\kappa, N)}\right)^{-1}\right\|_{2}^{-1} \geq \frac{\kappa_{I}}{|\kappa|} \min \left(1, \frac{1}{|\kappa|}\right) \frac{\pi}{5} h^{3} .
$$

(iv) If $\kappa_{R} \neq 0, \kappa_{I}=0$ then

$$
\left\|\left(A^{(\kappa, N)}\right)^{-1}\right\|_{2}^{-1} \geq \min \left(1, \frac{1}{\left|\kappa_{R}\right|}\right) \frac{\pi}{5 \sqrt{2}} h^{3} .
$$

Pr o of. By [16, Lemma 3.1] the Fourier transform of $\Phi(x, \kappa)$ is given by

$$
\widehat{\Phi}(\xi, \kappa)=\frac{2 \pi}{\sqrt{|\xi|^{2}-\kappa^{2}}}
$$

and is holomorphic with respect to $z=|\xi|$, with branch points at $z= \pm \kappa$; moreover, it has positive real part for $z \in \mathbb{R}, \kappa_{I}>0$. 
By Lemma 4.1 we have for $\kappa_{I}>0$

$$
\left\|\left(A^{(\kappa, N)}\right)^{-1}\right\|_{2}^{-1} \geq \inf _{\Sigma_{k \in \omega^{N, 0}}\left|\alpha_{k}\right|^{2} \neq 0} \frac{\left|\sum_{k, k^{\prime} \in \omega^{N, 0}} \alpha_{k} A_{k, k^{\prime}}^{(\kappa, N)} \alpha_{k^{\prime}}\right|}{\sum_{k \in \omega^{N, 0}}\left|\alpha_{k}\right|^{2}} .
$$

By application of Plancherel's formula to (2.8) twice and taking into account the formula for $\widehat{\Phi}$, we obtain, with $v_{h}=\sum_{k \in \omega^{N, 0}} \alpha_{k} \varphi_{k}^{N, 0}$ and $S^{h, 0}=\operatorname{span}\left\{\varphi_{k}^{N, 0} \mid\right.$ $\left.k \in \omega^{N, 0}\right\}$,

$$
\begin{aligned}
\left\|\left(A^{(\kappa, N)}\right)^{-1}\right\|_{2}^{-1} & \geq \inf _{v_{h} \neq 0, v_{h} \in S^{h, 0}} \frac{4 \pi h^{2}\left(V_{\kappa} v_{h}, v_{h}\right)}{\left\langle v_{h}, v_{h}\right\rangle_{0}} \\
& =2 \pi h^{2} \inf _{v_{h} \neq 0, v_{h} \in S^{h, 0}} \frac{\int_{\mathbb{R}^{2}}\left(\sqrt{|\xi|^{2}-\kappa^{2}}\right)^{-1}\left|\widehat{v}_{h}(\xi)\right|^{2} d \xi}{\left\|v_{h}\right\|_{0}^{2}}
\end{aligned}
$$

The latter term was estimated in [18] for the case $\kappa=i$. Next, we reduce the case of arbitrary $\kappa$ to the known one $\kappa=i$.

If $\kappa_{I}>0$ the following inequality is obtained from [13, Lemma 5, (4.2)] (under a slightly different definition of the square root defining $\widehat{\Phi})$ :

$$
\operatorname{Re} \sqrt{|\xi|^{2}-\kappa^{2}} \geq \frac{\kappa_{I}}{|\kappa|} \sqrt{|\xi|^{2}+|\kappa|^{2}}
$$

From the estimate above and the triangle inequality we obtain

$$
\operatorname{Re} \frac{1}{\sqrt{|\xi|^{2}-\kappa^{2}}} \geq \frac{\kappa_{I}}{|\kappa|} \frac{1}{\sqrt{|\xi|^{2}+|\kappa|^{2}}} \geq \frac{\kappa_{I}}{|\kappa|} \min \left(1, \frac{1}{|\kappa|}\right) \frac{1}{\sqrt{|\xi|^{2}+1}} .
$$

So for $\kappa_{I}>0$ we proved

$$
\begin{aligned}
\left\|\left(A^{(\kappa, N)}\right)^{-1}\right\|_{2}^{-1} & \geq 2 \pi h^{2} \frac{\kappa_{I}}{|\kappa|} \min \left(1, \frac{1}{|\kappa|}\right) \inf _{v_{h} \neq 0, v_{h} \in S^{h, 0}} \frac{\int_{\mathbb{R}^{2}} \frac{\left|\hat{v}_{h}(\xi)\right|^{2}}{\sqrt{|\xi|^{2}+1}} d \xi}{\left\|v_{h}\right\|_{0}^{2}} \\
& =2 \pi h^{2} \frac{\kappa_{I}}{|\kappa|} \min \left(1, \frac{1}{|\kappa|}\right) \inf _{v_{h} \neq 0, v_{h} \in S^{h, 0}} \frac{\left\|v_{h}\right\|_{\widetilde{H}^{-1 / 2}\left([-1,1]^{2}\right)}^{2}}{\left\|v_{h}\right\|_{0}^{2}} .
\end{aligned}
$$

The last term can be estimated when a constant $C$ is determined such that the inverse inequality

$$
\left\|v_{h}\right\|_{0} \leq \frac{C}{\sqrt{h}}\left\|v_{h}\right\|_{\widetilde{H}^{-1 / 2}[-1,1]^{2}} \quad\left(v_{h} \in S^{h, 0}\right)
$$

holds. In [18, Theorem 4.5, Corollary 4.1] it was proved by rough estimates that the constant can be chosen to be $\sqrt{10}$. So in the case $\kappa_{I}>0$ we showed that the closure of the numerical range of $A^{(\kappa, N)}$, defined by $\left\{\left(A^{(\kappa, N)} x, x\right) /(x, x) \mid\right.$ $\left.x \in \mathbb{C}^{n} \backslash\{0\}\right\}$, does not contain zero for any $N \in \mathbb{N}$, which implies invertibility of these matrices, stated in part (i) of the theorem. Furthermore, collecting the above inequalities we have proved (4.3). 
(4.2) was proved in [18]; it remains to prove statement (iv) of the theorem. We define $\sqrt{|\xi|^{2}-\kappa_{R}^{2}}$ so that it has real and positive values if $|\xi|^{2}>\kappa_{R}^{2}$ and we define it by $i \sqrt{\left.|| \xi\right|^{2}-\kappa_{R}^{2} \mid}$ if $|\xi|^{2}<\kappa_{R}^{2}$. Then it follows with $v_{h} \in S^{h, 0}$ that

$$
\begin{aligned}
\left|\int_{\mathbb{R}^{2}} \frac{\left|\widehat{v}_{h}(\xi)\right|^{2}}{\sqrt{|\xi|^{2}-\kappa_{R}^{2}}} d \xi\right| & =\frac{1}{\sqrt{2}}\left(\left|\int_{\mathbb{R}^{2}} \operatorname{Re} \frac{\left|\widehat{v}_{h}(\xi)\right|^{2}}{\sqrt{|\xi|^{2}-\kappa_{R}^{2}}} d \xi\right|+\left|\int_{\mathbb{R}^{2}} \operatorname{Im} \frac{\left|\widehat{v}_{h}(\xi)\right|^{2}}{\sqrt{|\xi|^{2}-\kappa_{R}^{2}}} d \xi\right|\right) \\
& \geq \frac{1}{\sqrt{2}}\left(\int_{|\xi|^{2}>\kappa_{R}^{2}} \frac{\left|\widehat{v}_{h}(\xi)\right|^{2}}{\sqrt{|\xi|^{2}-\kappa_{R}^{2}}} d \xi+\int_{|\xi|^{2}<\kappa_{R}^{2}} \frac{\left|\widehat{v}_{h}(\xi)\right|^{2}}{\sqrt{\left.|| \xi\right|^{2}-\kappa_{R}^{2} \mid}} d \xi\right) \\
& \geq \frac{1}{\sqrt{2}} \min \left(1, \frac{1}{\left|\kappa_{R}\right|}\right) \int_{\mathbb{R}^{2}} \frac{\left|\widehat{v}_{h}(\xi)\right|^{2}}{\sqrt{|\xi|^{2}+1}} d \xi
\end{aligned}
$$

The rest of the proof of (4.4) runs along the lines of the proof of (iii).

We shall use Banach's fixed point principle in finite-dimensional spaces to prove invertibility criteria for the matrices defined by the discretized Galerkin method. The following result is well known:

LEMMA 4.3. If

$$
\left\|A^{(\kappa, N)}-q^{\kappa, L, M, N}\right\|_{\infty}<\left\|\left(A^{(\kappa, N)}\right)^{-1}\right\|_{2}^{-1},
$$

then the matrix $q^{\kappa, L, M, N}$ is invertible.

We now state sufficient conditions for invertibility of our discretized Galerkin and collocation schemes.

THEOREM 4.4. (i) If $L \geq 3$ and $M \geq 3$ the matrices $q^{0, L, M, N}$ are invertible for all $N \in \mathbb{N}$.

(ii) If $\kappa_{I} \geq 0$ then there exist integers $L(\kappa), M(\kappa)$ such that there is an integer $N(\kappa)$ such that for all $L, M, N \in \mathbb{N}, L \geq L(\kappa), M \geq M(\kappa), N \geq N(\kappa)$ the matrices $q^{\kappa, L, M, N}$ are invertible.

Proof of (i). In Theorem 3.5 we proved

$$
\left\|A^{(0, N)}-q^{0, L, M, N}\right\|_{\infty} \leq h^{3}\left(\frac{1}{2(L-1)^{2}}+\frac{\pi}{6(L-1)}\right)+5.1 \frac{h^{3}}{M^{4}} .
$$

By (4.2) and Lemma 4.3, to guarantee the invertibility of $q^{0, L, M, N}$ it is sufficient to find $L$ and $M$ such that

$$
h^{3}\left(\frac{1}{2(L-1)^{2}}+\frac{\pi}{6(L-1)}\right)+5.1 \frac{h^{3}}{M^{4}}<\frac{\pi}{5} h^{3} .
$$

It is easily checked that one can take $L=3=M$. The proof of (ii) is analogous.

Remark. The factor of $h^{4}$ in equation (3.10) grows with $L^{2}$, so that one should choose $L$ as small as possible to ensure stability for not too small numbers $h$. 
5. Error estimates. We shall present a result on quasi-optimal convergence of the discretized Galerkin and collocation methods. We need the following preparatory lemma.

LEMMA 5.1. If there are constants $0<C_{1}<C_{2}$ such that the sequences of matrices $q^{\kappa, L, M, N}$ and $A^{(\kappa, N)}$ satisfy

$$
\left\|A^{(\kappa, N)}-q^{\kappa, L, M, N}\right\|_{\infty} \leq C_{1} h^{3}
$$

and

$$
\inf _{0 \neq x \in \mathbb{C}^{4 N^{2}}} \frac{\left|\left(A^{(\kappa, N)} x, x\right)\right|}{\|x\|^{2}}>C_{2} h^{3}
$$

(here $4 N^{2}$ is the number of elements in $\omega^{N, 0}$ ), then for all $\varepsilon>0$ and for all $x \in \mathbb{C}^{4 N^{2}}$,

$$
\left|\left(A^{(\kappa, N)} x, x\right)\right| \leq\left(\frac{C_{2}}{C_{2}-C_{1}}+\varepsilon\right)\left|\left(q^{(\kappa, L, M, N)} x, x\right)\right| .
$$

Proof. We estimate the bilinear forms of the matrices in question for any vector $x \in \mathbb{C}^{4 N^{2}}$ :

$$
\begin{aligned}
&\left|\left(\left(\frac{C_{2}}{C_{2}-C_{1}}+\varepsilon\right) q^{(\kappa, L, M, N)} x, x\right)\right|-\left|\left(A^{(\kappa, N)} x, x\right)\right| \\
&=\mid\left(\frac{C_{2}}{C_{2}-C_{1}}+\varepsilon\right)\left(\left(q^{(\kappa, L, M, N)}-A^{(\kappa, N)}\right) x, x\right) \\
&+\left(\frac{C_{2}}{C_{2}-C_{1}}+\varepsilon\right)\left(A^{(\kappa, N)} x, x\right)-\left(A^{(\kappa, N)} x, x\right) \mid \\
& \geq\left|\left(\frac{C_{2}}{C_{2}-C_{1}}+\varepsilon-1\right)\left(A^{(\kappa, N)} x, x\right)\right| \\
&-\left(\frac{C_{2}}{C_{2}-C_{1}}+\varepsilon\right)\left|\left(\left(q^{(\kappa, L, M, N)}-A^{(\kappa, N)}\right) x, x\right)\right| \\
& \geq\left|\left(\frac{C_{2}}{C_{2}-C_{1}}+\varepsilon-1\right)\left(A^{(\kappa, N)} x, x\right)\right| \\
&-\left(\frac{C_{2}}{C_{2}-C_{1}}+\varepsilon\right)\left\|q^{(\kappa, L, M, N)}-A^{(\kappa, N)}\right\|_{2}(x, x) \\
& \geq\left(\left(\frac{C_{2}}{C_{2}-C_{1}}+\varepsilon-1\right) C_{2} h^{3}-\left(\frac{C_{2}}{C_{2}-C_{1}}+\varepsilon\right) C_{1} h^{3}\right)(x, x) \\
&= \varepsilon h^{3}(x, x) \geq 0 . \text { - }
\end{aligned}
$$

Next we present our main result:

THEOREM 5.2. There are integers $L(\kappa), M(\kappa)$ such that the discretized Galerkin equations (2.17) and the discretized collocation equations (2.18) are uniquely 
solvable for sufficiently large $N$ and there is a constant $C>0$ such that for any function $f \in H^{2+\varepsilon}(\Omega)$ and any $0<\varepsilon<\frac{1}{2}$ we have the error estimates

$$
\begin{aligned}
\left\|u^{\kappa}-u_{N}^{\kappa}\right\|_{\widetilde{H}^{1 / 2}(\Omega)} & \leq C h^{1 / 2-\varepsilon}\|f\|_{H^{2+\varepsilon}(\Omega)}, \\
\left\|v^{\kappa}-v_{N}^{\kappa}\right\|_{\widetilde{H}^{1 / 2}(\Omega)} & \leq C h^{1 / 2-\varepsilon}\|f\|_{H^{2+\varepsilon}(\Omega)} .
\end{aligned}
$$

Proof. From the proof of Theorem 4.4 it follows that we can choose numbers $M(\kappa), L(\kappa)$ such that the assertions of Lemma 5.1 are satisfied for sufficiently large $N \geq N(\kappa)$. On the space $S^{h, 0}$ of piecewise constant functions $w_{h}=\sum_{k \in \omega^{N, 0}} \alpha_{k} \varphi_{k}^{N, 0}$ we define the sesquilinear form $\left(Q^{\kappa, L, M, N} w_{h}, w_{h}\right)$ associated with $q^{\kappa, L, M, N}$ by

$$
\left(Q^{\kappa, L, M, N} w_{h}, w_{h}\right):=\sum_{k, k^{\prime} \in \omega^{N, 0}} \alpha_{k} q_{k, k^{\prime}}^{\kappa, L, M, N} \overline{\alpha_{k^{\prime}}} .
$$

There is a constant $C_{1}>0$ such that the functions $u_{N}^{\kappa}=\sum_{k \in \omega^{N, 0}} \gamma_{k}^{\kappa} \varphi_{k}^{N, 0}$ defined by equation (2.17) and $u_{h}^{\kappa}=\sum_{k \in \omega^{N, 0}} \alpha_{k}^{\kappa} \varphi_{k}^{N, 0}$ defined by (2.5) satisfy

$$
\begin{aligned}
\left\|u_{N}^{\kappa}-u_{h}^{\kappa}\right\|_{\widetilde{H}^{-1 / 2}(\Omega)}^{2} & \leq 2 \pi\left(V_{\kappa}\left(u_{h}^{\kappa}-u_{N}^{\kappa}\right), u_{h}^{\kappa}-u_{N}^{\kappa}\right) \\
& \leq C_{1}\left(Q^{\kappa, L(\kappa), M(\kappa), N}\left(u_{h}^{\kappa}-u_{N}^{\kappa}\right), u_{h}^{\kappa}-u_{N}^{\kappa}\right) .
\end{aligned}
$$

Using (2.5), (2.17) we obtain

$$
\begin{aligned}
\left(Q^{\kappa, L(\kappa), M(\kappa), N}\right. & \left.\left(u_{h}^{\kappa}-u_{N}^{\kappa}\right), u_{h}^{\kappa}-u_{N}^{\kappa}\right) \\
= & \left(Q^{\kappa, L(\kappa), M(\kappa), N}\left(u_{h}^{\kappa}-u_{N}^{\kappa}\right), u_{h}^{\kappa}\right)-\sum_{k \in \omega^{N, 0}}\left(\alpha_{k}^{\kappa}-\gamma_{k}^{\kappa}\right) N^{2} \overline{f\left(x_{k}\right)} \\
= & \left(Q^{\kappa, L(\kappa), M(\kappa), N}\left(u_{h}^{\kappa}-u_{N}^{\kappa}\right), u_{h}^{\kappa}\right)-\left(V_{\kappa}\left(u_{h}^{\kappa}-u_{N}^{\kappa}\right), u_{h}^{\kappa}\right) \\
& +\left(f, u_{h}^{\kappa}-u_{N}^{\kappa}\right)-\sum_{k \in \omega^{N, 0}}\left(\left(\alpha_{k}^{\kappa}-\gamma_{k}^{\kappa}\right) N^{2} \overline{f\left(x_{k}\right)}\right) .
\end{aligned}
$$

We estimate the first term as in [18]:

$$
\begin{aligned}
\mid\left(Q^{\kappa, L(\kappa), M(\kappa), N}\right. & \left.\left(u_{h}^{\kappa}, u_{h}^{\kappa}-u_{N}^{\kappa}\right)-\left(V u_{h}^{\kappa}, u_{h}^{\kappa}-u_{N}^{\kappa}\right)\right) \mid \\
& \leq C_{2}\left\|A^{(\kappa, N)}-Q^{\kappa, L(\kappa), M(\kappa), N}\right\|_{2}\left\|u_{h}^{\kappa}\right\|_{0}\left\|u_{h}^{\kappa}-u_{N}^{\kappa}\right\|_{0} h^{-2} \\
& \leq C_{2}\left\|A^{(\kappa, N)}-Q^{\kappa, L(\kappa), M(\kappa), N}\right\|_{\infty}\left\|u_{h}^{\kappa}\right\|_{0}\left\|u_{h}^{\kappa}-u_{N}^{\kappa}\right\|_{0} h^{-2} \\
& \leq C_{3} h^{3}\left\|u_{h}^{\kappa}-u_{N}^{\kappa}\right\|_{\widetilde{H}^{-1 / 2}(\Omega)} h^{-1 / 2}\left\|u_{h}^{\kappa}\right\|_{H^{-\varepsilon}(\Omega)} h^{-\varepsilon} h^{-2} .
\end{aligned}
$$

This holds with constants $C_{2}, C_{3}$ independent of $h$. Here we used again the equivalence (up to the factor of $h$ ) of the $L^{2}$-norm to its discrete version and the inverse inequality. By these methods, the Cauchy-Schwarz inequality and the result in [5, Lemma 3.7], which carries over to piecewise constant trial functions, we get, with other constants $C_{4}, C_{5}$, 


$$
\begin{aligned}
\mid\left(f, u_{h}^{\kappa}-u_{N}^{\kappa}\right)- & \sum_{k \in \omega^{N, 0}}\left(\left(\alpha_{k}^{\kappa}-\gamma_{k}^{\kappa}\right) N^{2} \overline{f\left(x_{k}\right)}\right) \mid \\
& \leq \sqrt{\sum_{k \in \omega^{N, 0}}\left|\alpha_{k}^{\kappa}-\gamma_{k}^{\kappa}\right|^{2}} \sqrt{\sum_{k \in \omega^{N, 0}}\left|\left(\varphi_{k}^{N, 0}, f\right)-N^{2} f\left(x_{k}^{N}\right)\right|^{2}} \\
& \leq C_{4} h^{-1}\left\|u_{h}^{\kappa}-u_{N}^{\kappa}\right\|_{0}\|f\|_{H^{2+\varepsilon}(\Omega)} h^{2} \\
& \leq C_{5} h^{1 / 2}\left\|u_{h}^{\kappa}-u_{N}^{\kappa}\right\|_{\widetilde{H}^{-1 / 2}(\Omega)}\|f\|_{H^{2+\varepsilon}(\Omega)} .
\end{aligned}
$$

So we have proved

$$
\begin{aligned}
\left\|u_{N}^{\kappa}-u_{h}^{\kappa}\right\|_{\widetilde{H}^{-1 / 2}(\Omega)}^{2} & \leq C_{3} h^{1 / 2-\varepsilon}\left\|u_{h}^{\kappa}-u_{N}^{\kappa}\right\|_{\widetilde{H}^{-1 / 2}(\Omega)}\left\|u_{h}^{\kappa}\right\|_{H^{-\varepsilon}(\Omega)} \\
& +C_{5} h^{1 / 2}\left\|u_{h}^{\kappa}-u_{N}^{\kappa}\right\|_{\widetilde{H}^{-1 / 2}(\Omega)}\|f\|_{H^{2+\varepsilon}(\Omega)} .
\end{aligned}
$$

We divide this inequality by $\left\|u_{h}^{\kappa}-u_{N}^{\kappa}\right\|_{\widetilde{H}^{-1 / 2}(\Omega)}$ to get

$$
\left\|u_{h}^{\kappa}-u_{N}^{\kappa}\right\|_{\widetilde{H}^{-1 / 2}(\Omega)} \leq C_{3} h^{1 / 2-\varepsilon}\left\|u_{h}^{\kappa}\right\|_{H^{-\varepsilon}(\Omega)}+C_{5} h^{1 / 2}\|f\|_{H^{2+\varepsilon}(\Omega)} .
$$

By a standard trick $[26],[18$, p. 16], $[5$, p. 12] it can be proved that there is a constant $C_{6}$ such that

$$
\left\|u_{h}^{\kappa}\right\|_{H^{-\varepsilon}(\Omega)} \leq C_{6}\left\|u^{\kappa}\right\|_{H^{-\varepsilon}(\Omega)} .
$$

Because of the invertibility of $V_{\kappa}: H^{\varepsilon}(\Omega) \rightarrow H^{1+\varepsilon}(\Omega)$ and the continuous imbedding of $H^{2+\varepsilon}(\Omega)$ into $H^{1+\varepsilon}(\Omega)$ there is a constant $C_{7}$ such that

$$
\left\|u_{h}^{\kappa}\right\|_{H^{-\varepsilon}(\Omega)} \leq C_{7}\|f\|_{H^{2+\varepsilon}(\Omega)} .
$$

Collecting of all the estimates we obtain (5.4).

On the space $S^{h, 1}$ of piecewise bilinear functions $z_{h}=\sum_{k \in \omega^{N, 1}} \beta_{k} \varphi_{k}^{N, 1}$ we define the bilinear form $\left(Q_{c}^{\kappa, L, M, N} z_{h}, z_{h}\right)$ associated with $q^{\kappa, L, M, N}$ by

$$
\left(Q_{c}^{\kappa, L, M, N} z_{h}, z_{h}\right):=\sum_{k, k^{\prime} \in \omega^{N, 1}} \beta_{k} q_{k, k^{\prime}}^{\kappa, L, N} \overline{\beta_{k^{\prime}}} .
$$

The proof of the convergence of the discretized collocation solutions runs along the same lines as the one for the discretized Galerkin solutions. The essential fact we need is the convergence of the collocation solutions $v_{h}^{\kappa}$ defined by (2.6), which is proved in [4] (see also [5]). In particular, the existence of a constant $C_{8}>0$ such that

$$
\left|\left(V_{\kappa} z_{h}, z_{h}\right)\right| \leq C_{8} h^{2} \sum_{k, k^{\prime} \in \omega^{N, 1}} \beta_{k}\left(V_{\kappa} \varphi_{k}^{N, 1}\right)\left(x_{k^{\prime}}^{N, 1}\right) \overline{\beta_{k^{\prime}}}
$$

holds for all $z_{h}=\sum_{k \in \omega^{N, 1}} \beta_{k} \varphi_{k}^{N, 1} \in S^{h, 1}$ was obtained in [4, 5]. From this and Lemma 5.1 we obtain the estimate

$$
\left\|v_{h}^{\kappa}-v_{N}^{\kappa}\right\|_{\widetilde{H}^{1 / 2}(\Omega)}^{2} \leq C_{8}\left|\left(Q_{c}^{\kappa, L, M, N}\left(v_{h}^{\kappa}-v_{N}^{\kappa}\right), v_{h}^{\kappa}-v_{N}^{\kappa}\right)\right| .
$$


From (2.6) and (2.18) we obtain a constant $C_{9}$ such that

$$
\begin{aligned}
& \left|\left(Q_{c}^{\kappa, L, M, N}\left(v_{h}^{\kappa}-v_{N}^{\kappa}\right), v_{h}^{\kappa}-v_{N}^{\kappa}\right)\right| \\
& \quad=\left|\left(Q_{c}^{\kappa, L, M, N}\left(v_{h}^{\kappa}-v_{N}^{\kappa}\right), v_{h}^{\kappa}\right)-\sum_{k, k^{\prime} \in \omega^{N, 1}}\left(\beta_{k}^{\kappa}-\varrho_{k}^{\kappa}\right) h^{2}\left(V_{\kappa} \varphi_{k}^{N, 1}\right)\left(x_{k^{\prime}}^{N, 1}\right) \overline{\varrho_{k^{\prime}}}\right| \\
& \quad \leq C_{9}\left\|v_{h}^{\kappa}-v_{N}^{\kappa}\right\|_{\widetilde{H}^{-1 / 2}(\Omega)}\left\|v_{h}^{\kappa}\right\|_{H^{-\varepsilon}(\Omega)},
\end{aligned}
$$

taking into account the consistency of the matrices in the last inequality. By the same trick we used above, we finally obtain (5.5).

6. Numerical results. Some results for the capacity of the square plate $[-1,1]^{2}$ found by our discretized Galerkin and collocation methods are presented. We have chosen the parameters to be $L=3=M$. Let us point out that our aim was not to improve the methods to approximate the true solution. Indeed, our results are worse than the results given in $[11,12,20]$ found by mesh refinement. Our main interest was to state and prove error estimates for the discretized Galerkin and collocation methods by use of the midpoint formula, which reduces computational work.

In the following tables we list, in the first column, the number $N$ of partitions of $[0,1]$, in the second column the capacity $C_{N}^{0}:=\int_{[-1,1]^{2}} u_{N}^{(0)}(x) d x$ determined by our discretized Galerkin method, in the third column we present the values $C_{N}$ we derived in [18] by a discretized Galerkin method of high accuracy. The error terms we gave in brackets stem from analytical a posteriori results and determine the intervals where the capacities determined by the true Galerkin solutions are. In the fourth column we list the experimental convergence rate $\alpha$, which is defined by

$$
\alpha:=\ln \frac{0.7335-C_{N}^{0}}{0.7335-C_{N+1}^{0}}\left(\ln \frac{N+1}{N}\right)^{-1}
$$

Here we use the extrapolated value 0.7335 for the capacity used in $[11,12,20]$. The theoretical value for this convergence rate is $1-\varepsilon$. The first table was calculated by discretized Galerkin's method using piecewise constant elements.

\begin{tabular}{|c|c|c|c|}
\hline$N$ & $C_{N}^{0}$ & $C_{N}$ & $\alpha$ \\
\hline 1 & 0.67266 & $0.67267 \pm 0.00064$ & - \\
\hline 2 & 0.69995 & $0.69995 \pm 0.00041$ & 0.85 \\
\hline 3 & 0.71042 & $0.70993 \pm 0.00030$ & 0.92 \\
\hline 4 & 0.71595 & $0.71531 \pm 0.00024$ & 0.95 \\
\hline 5 & 0.71937 & $0.71868 \pm 0.00020$ & 0.97 \\
\hline 6 & 0.72169 & $0.72099 \pm 0.00018$ & 0.98 \\
\hline 7 & 0.72337 & $0.72268 \pm 0.00016$ & 0.99 \\
\hline 8 & 0.72464 & $0.72396 \pm 0.00014$ & 1.00 \\
\hline
\end{tabular}


In the next table we calculated $C_{N, c}=\int_{[-1,1]^{2}} v_{N}^{(0)}(x) d x$ determined by our discretized collocation method with piecewise bilinear trial functions collocating at the nodes.

\begin{tabular}{|c|c|c|}
\hline$N$ & $C_{N, c}$ & $\alpha$ \\
\hline 1 & 0.33633 & - \\
\hline 2 & 0.51846 & 0.88 \\
\hline 3 & 0.58851 & 0.97 \\
\hline 4 & 0.62436 & 0.98 \\
\hline 5 & 0.64605 & 0.99 \\
\hline 6 & 0.66057 & 1.00 \\
\hline 7 & 0.67097 & 1.00 \\
\hline 8 & 0.67879 & 1.00 \\
\hline
\end{tabular}

\section{References}

[1] D. Colton and R. Kress, Integral Equation Methods in Scattering Theory, Wiley, 1983.

[2] M. Costabel, Boundary integral operators on Lipschitz domains: Elementary results, SIAM J. Math. Anal. 19 (1988), 613-626.

[3] M. Costabel and W. McLean, Spline collocation for strongly elliptic equations on the torus, Numer. Math. 62 (1992), 511-538.

[4] M. Costabel, F. Penzel and R. Schneider, Error analysis of a boundary element collocation method for a screen problem in $\mathbb{R}^{3}$, Math. Comp. 58 (198) (1992), 575-586.

[5] - , - - , Convergence of boundary element collocation methods for Dirichlet and Neumann screen problems in $\mathbb{R}^{3}$, Appl. Anal. 49 (1993), 101-117.

[6] M. Costabel and E. Stephan, Duality estimates for the numerical solution of integral equations, Numer. Math. 54 (1988), 339-353.

[7] M. Dauge, Elliptic Boundary Value Problems in Corner Domains - Smoothness and Asymptotics of Solutions, Lecture Notes in Math. 1341, Springer, Berlin 1988.

[8] P. J. Davis and P. Rabinowitz, Methods of Numerical Integration, Academic Press, New York 1975

[9] M. G. Duffy, Quadrature over a pyramid or cube of integrands with a singularity at a vertex, SIAM J. Numer. Anal. 19 (6) (1982), 1260-1262.

[10] V. J. Ervin and E. P. Stephan, Experimental convergence of boundary element methods for the capacity of the electrified plate, Boundary Elem. 9 (1987), 167-175.

[11] —, - A boundary-element method with mesh refinement for a weakly singular integral equation, Comm. Appl. Numer. Methods 7 (4) (1991), 273-280.

[12] V. J. Ervin, E. P. Stephan and S. Abou el-Seoud, An improved boundary element method for the charge density of a thin electrified plate in $\mathbb{R}^{3}$, Math. Methods Appl. Sci. 13 (1990), 291-303.

[13] T. Ha Duong, On the transient acoustic scattering by a flat object, Japan J. Appl. Math. 7 (3) (1990), 489-513.

[14] G. C. Hsiao, P. Kopp and W. L. Wendland, A Galerkin collocation method for some integral equations of the first kind, Computing 25 (1980), 89-130.

[15] G. C. Hsiao and W. L. Wendland, A finite element method for some integral equations of the first kind, J. Math. Anal. Appl. 58 (1977), 449-481. 
[16] R. C. Mac Camy and E. Stephan, Solution procedures for three-dimensional eddy current problems, ibid. 101 (1984), 348-379.

[17] J. C. Nedelec and J. Planchard, Une méthode variationnelle d'éléments finis pour la résolution numérique d'un problème extérieur dans $\mathbb{R}^{3}$, RAIRO 7 (1973), 105-129.

[18] F. Penzel, Error estimates for a discretized Galerkin method for a boundary integral equation in two dimensions, Numer. Methods Partial Differential Equations 8 (1992), 405-421.

[19] - Error estimates for discretized Galerkin and collocation boundary element methods for time harmonic Dirichlet screen problems in $\mathbb{R}^{3}$, THD-Preprint, Technische Hochschule Darmstadt, 1991.

[20] T. v. Petersdorff, Randwertprobleme der Elastizitätstheorie für Polyeder-Singularitäten und Approximation mit Randelementmethoden, Dissertation, Darmstadt 1989.

[21] S. Prössdorf, Numerische Behandlung singulärer Integralgleichungen, Z. Angew. Math. Mech. 69 (1989), T5-T13.

[22] S. Prössdorf and A. Rathsfeld, Quadrature and Collocation methods for singular integral equations on curves with corners, Z. Anal. Anwendungen 8 (1989), 197-220.

[23] S. Prössdorf and R. Schneider, A spline collocation method for multidimensional strongly elliptic pseudodifferential operators of order zero, Integral Equations Operator Theory 14 (1991), 399-435.

[24] -, -, Spline approximation methods for multidimensional periodic pseudodifferential equations, Integral Equations Operator Theory 15 (1992), 626-672.

[25] A. Rathsfeld, On the stability of quadrature methods for the double layer potential equation over the boundary of the polyhedron, manuscript, May 1991.

[26] G. Schmidt, Splines und die näherungsweise Lösung von Pseudodifferentialgleichungen auf geschlossenen Kurven, report R-MATH-09/86, Karl-Weierstrass-Institut, Berlin 1986

[27] R. Schneider, Stability of a spline collocation method for strongly elliptic multidimensional singular integral equations, Numer. Math. 58 (1991), 855-873.

[28] C. Schwab and W. L. Wendland, On numerical cubatures of singular surface integrals in boundary element methods, ibid. 62 (1992), 343-369.

[29] E. Stephan, Boundary integral equations for screen problems in $\mathbb{R}^{3}$, Integral Equations Operator Theory 10 (1987), 236-257.

[30] A. H. Strout and D. Secrest, Gaussian Quadrature Formulas, Prentice-Hall, 1966.

[31] W. L. Wendland, Boundary element methods and their asymptotic convergence, in: Lecture Notes of the CISM Summer School on "Theoretical Acoustics and Numerical Techniques", Internat. Centre for Mechanical Sciences, Udine, Italy, 1983, P. Filippi (ed.), Lecture Notes in Phys., Springer, 135-216.

[32] W. L. Wendland, Strongly elliptic boundary integral equations, in: The State of the Art in Numerical Analysis, A. Iserles and M. J. D. Powell (eds.), Clarendon Press, Oxford $1987,511-562$. 